\title{
Three-dimensional magnetic reconnection through a moving magnetic null
}

\author{
V. S. Lukin and M. G. Linton \\ Space Science Division, Naval Research Laboratory, Washington, DC 20375, USA
}

Received: 12 August 2011 - Revised: 14 November 2011 - Accepted: 16 November 2011 - Published: 28 November 2011

\begin{abstract}
A computational study of three-dimensional magnetic reconnection between two flux ropes through a moving reconnection site is presented. The configuration is considered in the context of two interacting spheromaks constrained by a perfectly conducting cylindrical boundary and oriented to form a single magnetic field null at its center. The initial magnetic field configuration is embedded into a uniform thermal plasma and is unstable to tilting. As the spheromaks tilt, their magnetic fields begin to reconnect at the null, subsequently displacing both the null and the reconnection site. The motion of the reconnection region and the magnetic null are shown to be correlated, with stronger correlation and faster reconnection observed in plasmas with lower thermal to magnetic pressure ratio. It is also shown that ion inertial effects allow for yet faster reconnection, but do not qualitatively change the dynamics of the process. Implications of the coupling between moving magnetic nulls and reconnection sites, as well as of possible mechanisms for fast reconnection through a moving reconnection region, are discussed. The simulations are conducted using both single-fluid and Hall MHD plasma models within the HiFi multi-fluid modeling framework.
\end{abstract}

\section{Introduction}

Magnetic reconnection in laboratory, astrophysical, and idealized settings has been widely investigated in the past decade (Zweibel and Yamada, 2009). Much of the focus, particularly in the computational modeling community, has been on idealized two-dimensional (2-D) systems (Sweet, 1958; Parker, 1957; Birn et al., 2001; Bhattacharjee et al., 2005; Loureiro et al., 2005; Simakov and Chacón, 2008;

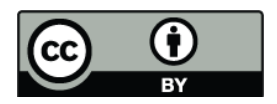

Correspondence to: V. S. Lukin (vlukin1@mailaps.org)
Daughton et al., 2009). There, the structure of a 2-D reconnection region (RR) and physical mechanisms that may allow for reconnection rates comparable to those observed or implied from space and astrophysical observations have been investigated. At the same time, important theoretical progress has been made in classifying and understanding the geometry of a globally three-dimensional (3-D) RR (Priest and Titov, 1996; Linton et al., 2001). In particular, the topology, ideal and non-ideal evolution of magnetic field lines in the neighborhood of an isolated stationary 3-D magnetic null have been extensively investigated (Lau and Finn, 1990; Pontin et al., 2007; Priest and Pontin, 2009); a recent review paper by Pontin (2011) provides a good set of examples of the past and ongoing work on this subject. These studies have focused primarily on null-point reconnection in the Earth's magneto-tail (Xiao et al., 2006) and solar (Fletcher et al., 2001; Maclean et al., 2009; Masson et al., 2009) contexts, though laboratory investigations of magnetic reconnection are also beginning to explore local and topological properties of quasi-stationary 3-D reconnection sites (Frank and Bogdanov, 2001; Cothran et al., 2003; Intrator et al., 2009; Lawrence and Gekelman, 2009).

The key distinguishing aspects of globally 3-D reconnection is the 3-D localization of the RR and the ability of the magnetic structure associated with the RR to extend and/or move in the direction normal to the reconnection plane, i.e. in the direction co- or counter-aligned with the reconnection electric field. Observational signatures of the RR localization (Munsat et al., 2007) and motion in solar flares (Qiu, 2009) and laboratory experiments (Katz et al., 2011) indicate that the reconnection magnetic structure can propagate at a substantial fraction of the local Alvén speed with respect to surrounding plasma, but there have been few numerical studies that have focused on the propagating RR phenomenon (e.g. Lapenta et al., 2006 and references therein). Some of the 3-D numerical studies have considered magnetic configurations with a pre-existing wide current sheet where a 3-D

Published by Copernicus Publications on behalf of the European Geosciences Union and the American Geophysical Union. 
$\mathrm{RR}$ is initiated by localized thinning of the current layer and formation of a short reconnection x-line. In kinetic, hybrid and Hall magnetohydrodynamic (MHD) simulations, such a RR has been observed to extend along the $x$-line in the direction of motion of the dominant current carriers (Lapenta et al., 2006); there appear to be no documented studies of a similar system using a single-fluid MHD model. On the other hand, previous single-fluid MHD simulations of magnetic configurations that include a 3-D magnetic null have noted motion, rather than an extension, of the associated RR (Pontin and Galsgaard, 2007; Masson et al., 2009), but have not explored this aspect of 3-D reconnection in detail.

In the present study, we investigate 3-D reconnection in a magnetic field configuration with no pre-existing current sheet and no global 2-D symmetry that could be utilized to analyze the resulting RR topology and dynamics. This work follows a previously published report of the first numerical simulation of 3-D null-point magnetic reconnection and dynamical merging of two simply-connected magnetic structures (spheromaks) validated by in-situ experimental observations (Gray et al., 2010). Here, a more detailed numerical study of the motion and structure of the 3-D RR as the spheromaks merge and their magnetic fields reconnect is presented. In particular, it is demonstrated that the motion of the RR is correlated with the motion of the null, and the correlation is enhanced in MHD plasmas with lower $\beta \equiv n k T /\left(B^{2} / 8 \pi\right)$. The lower $\beta$ simulations also show faster, more explosive onset of reconnection, while simulations where the ion inertial scale $\left(c / \omega_{p i}\right) \equiv c \sqrt{m_{i} / 4 \pi n e^{2}}$ becomes non-negligible relative to the smallest dimension of the RR allow for yet faster reconnection without qualitatively changing the dynamics of the process.

The manuscript is organized as follows. The partial differential equations (PDEs) being solved within the HiFi multi-fluid modeling framework (Glasser and Tang, 2004; Lukin, 2008; Meier et al., 2010; Lowrie et al., 2011; Lukin et al., 2011), the initial and boundary conditions, as well as other relevant details of the numerical model are described in Sect. 2. The global stability and evolution of the magnetic configuration, which initially consists of two identical spheromaks confined next to each other in a perfectlyconducting cylinder, is addressed in Sect. 3. The dynamics and structure of the magnetic null and the 3-D RR formed between the spheromaks are detailed in Sect. 4. Finally, general implications of the coupling between moving magnetic nulls and reconnection sites, as well as of possible mechanisms for fast reconnection through a moving reconnection region, are discussed in Sect. 5.

\section{Numerical model}

The simulations described below have been performed using the multi-fluid adaptive implicit spectral element HiFi framework (Lukin, 2008; Lukin et al., 2011), solving the following set of normalized compressible Hall MHD equations:

$$
\begin{gathered}
\frac{\partial \tilde{\rho}}{\partial \tilde{t}}+\tilde{\nabla} \cdot\left[\tilde{\rho} \tilde{\mathbf{v}}_{i}\right]=0 \\
\frac{\partial\left(\tilde{\rho} \tilde{\mathbf{v}}_{i}\right)}{\partial \tilde{t}}+\tilde{\nabla} \cdot\left[\tilde{\rho} \tilde{\mathbf{v}}_{i} \tilde{\mathbf{v}}_{i}+\tilde{p} \tilde{\mathbf{I}}-\mu_{i} \tilde{\nabla} \tilde{\mathbf{v}}_{i}-\mu_{e} \tilde{\nabla} \tilde{\mathbf{v}}_{e}\right]=\tilde{\mathbf{J}} \times \tilde{\mathbf{B}} \\
\tilde{\mathbf{E}}=-\frac{\partial \tilde{\mathbf{A}}}{\partial \tilde{t}}=-\tilde{\mathbf{v}}_{e} \times \tilde{\mathbf{B}}-\frac{d_{i}}{\tilde{\rho}} \tilde{\nabla} \tilde{p}_{e}+\mathbf{D}_{J} \\
\frac{3}{2} \frac{\partial \tilde{p}}{\partial \tilde{t}}+\tilde{\nabla} \cdot\left[\frac{5}{2}\left(\tilde{p}_{i} \tilde{\mathbf{v}}_{i}+\tilde{p}_{e} \tilde{\mathbf{v}}_{e}\right)-\kappa \tilde{\nabla} \tilde{T}\right] \\
=\tilde{\mathbf{v}}_{i} \cdot \tilde{\nabla} \tilde{p}_{i}+\tilde{\mathbf{v}}_{e} \cdot \tilde{\nabla} \tilde{p}_{e}+\mu_{i}\left\|\tilde{\nabla} \tilde{\mathbf{v}}_{i}\right\|^{2}+Q_{J}
\end{gathered}
$$

where

$$
\begin{aligned}
\tilde{\mathbf{B}} & =\tilde{\nabla} \times \tilde{\mathbf{A}}, \quad \tilde{\mathbf{J}}=\tilde{\nabla} \times \tilde{\mathbf{B}}=\tilde{\nabla}(\tilde{\nabla} \cdot \tilde{\mathbf{A}})-\tilde{\nabla}^{2} \tilde{\mathbf{A}} \\
\tilde{\mathbf{v}}_{e} & =\frac{\tilde{\rho} \tilde{\mathbf{v}}_{i}-d_{i} \tilde{\mathbf{J}}}{\tilde{\rho}}, \\
\tilde{p} & =\tilde{\rho} \tilde{T}=\tilde{p}_{i}+\tilde{p}_{e}, \quad \frac{\tilde{p}_{e}}{\tilde{p}_{i}}=\mathrm{const}, \\
\mathbf{D}_{J} & =\left\{\begin{array}{cc}
\left(d_{i} \mu_{e} / \tilde{\rho}\right) \tilde{\nabla}^{2} \tilde{\mathbf{v}}_{e}, & d_{i}>0 \\
-\nu \tilde{\nabla}^{2} \tilde{\mathbf{J}}, & d_{i}=0
\end{array},\right. \\
Q_{J} & =\left\{\begin{array}{cc}
\mu_{e}\left\|\tilde{\nabla} \tilde{\mathbf{v}}_{e}\right\|^{2}, & d_{i}>0 \\
\nu\|\tilde{\nabla} \tilde{\mathbf{J}}\|^{2}, & d_{i}=0
\end{array}\right.
\end{aligned}
$$

$\nabla=\left(1 / r_{0}\right) \tilde{\nabla}$ is the differential gradient operator, $t=t_{0} \tilde{t}$ is the time, $\rho=\rho_{0} \tilde{\rho}$ is the plasma density, $\mathbf{v}_{i, e}=v_{\mathrm{Alf}} \tilde{\mathbf{v}}_{i, e}$ are the ion and electron plasma velocities, $\mathbf{B}=B_{0} \tilde{\mathbf{B}}$ is the magnetic field, $\mathbf{E}=\left(v_{\mathrm{Alf}} B_{0} / c\right) \tilde{\mathbf{E}}$ is the electric field, $\mathbf{A}=r_{0} B_{0} \tilde{\mathbf{A}}$ is the electro-magnetic vector potential, $\mathbf{J}=\left(c B_{0} / 4 \pi r_{0}\right) \tilde{\mathbf{J}}$ is the current density, $p=p_{0} \tilde{p}$ and $T=\left(p_{0} / k n_{0}\right) \tilde{T}$ are the total thermal pressure and temperature of the plasma, and $p_{i, e}=p_{0} \tilde{p}_{i, e}$ are the ion and electron pressures with a constant and uniform relative ratio. Both independent and dependent variables have been normalized by choosing physical dimensions for distance $r_{0}$, plasma number density $n_{0}$, and magnetic induction $B_{0}$, expressing all other normalization units in terms of these three and the speed of light $c$ and Boltzmann's constant $k$. In particular, the plasma density is measured in units of $\rho_{0} \equiv m_{i} n_{0}$, the velocities are measured in units of Alfvén velocity $v_{\text {Alf }} \equiv B_{0} / \sqrt{4 \pi \rho_{0}}$, time is measured in units of $t_{0} \equiv r_{0} / v_{\text {Alf }}$, and plasma pressure is measured in units of $p_{0} \equiv B_{0}^{2} / 4 \pi$. The ion inertial scale has been normalized by the global system size $d_{i} \equiv\left(c / \omega_{p i}\right) / r_{0}$, $\kappa$ is the normalized heat conduction coefficient, $\mu_{i}$ and $\mu_{e}$ are the normalized viscosity coefficients for ion and electron flow, and $v$ is the normalized hyper-resistive coefficient providing magnetic dissipation in the limit of $d_{i} \rightarrow 0$. In writing the Hall MHD PDEs in this form, the Weyl gauge has been chosen by explicitly setting the electrostatic potential to zero and absorbing any electrostatic $\mathbf{E}$-field that may arise into the vector potential $\mathbf{A}$.

The solution of the Hall MHD PDEs is advanced in time using the fully implicit Crank-Nicholson scheme with 


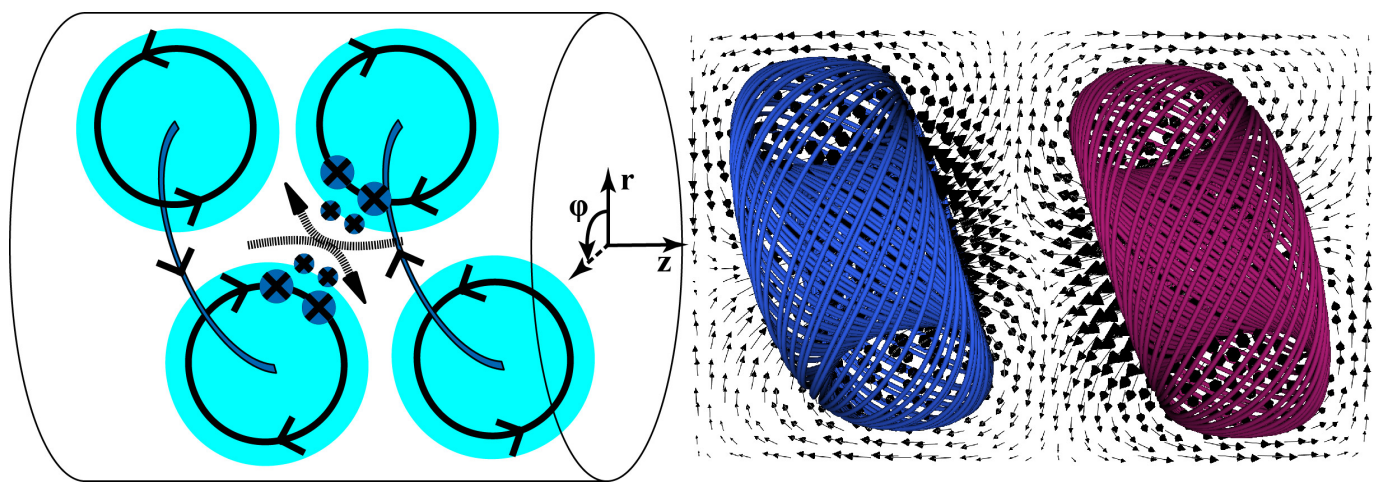

Fig. 1. A cartoon (left panel) and HiFi simulation (right panel) of two tilting spheromaks undergoing magnetic reconnection at the central magnetic null. The cartoon indicates the reconnecting in-plane B-field components and the co-directed out-of-plane B-field being convected into the RR. The simulation panel shows streamlines of two separate magnetic field-lines, and arrows show the magnetic field direction and strength at the mid-plane. Note that the two spheromaks have oppositely directed toroidal magnetic fields.

no time-step splitting (Glasser and Tang, 2004; Lukin, 2008). The computational domain geometry is a cylinder $\Omega(r, \phi, z)=\left[0, r_{0}\right] \times[0,2 \pi] \times[0, L]$ with $L=3 r_{0}$. The domain is discretized in space using $\left(n_{r}, n_{\phi}, n_{z}\right)=(16,30,48)$ elements in $\hat{r}, \hat{\phi}$, and $\hat{z}$ directions, respectively, where the $3^{r d}$ order modified Jacobi polynomials (Lukin, 2008; Lukin et al., 2011) are used to expand the solution in each direction within each element. Thus, the effective spatial resolution is $\left(N_{r}, N_{\phi}, N_{z}\right)=(48,90,144)$. The domain is periodic in $\phi$, with the following impenetrable, free-slip, perfect conductor boundary conditions imposed on the PDEs at $r=r_{0}, z=0$ and $z=L: \hat{n} \cdot \mathbf{v}_{i}=0,(\hat{n} \cdot \nabla)\left(\hat{n} \times \mathbf{v}_{i, e}\right)=\mathbf{0}, \partial(\hat{n} \times \mathbf{A}) / \partial t=\mathbf{0}$ and $\nabla \cdot \mathbf{A}=0$, where $\hat{n}$ is the unit normal vector at the boundary surface. These are supplemented with the fixed wall temperature condition $\left.T\right|_{\text {bound }}=\left.T\right|_{t=0}=$ const and the condition $\mu_{e} \hat{n} \cdot \nabla\left(\hat{n} \cdot \mathbf{v}_{e}\right)=0$ [or $\nu \hat{n} \cdot \nabla(\hat{n} \cdot \mathbf{J})=0$, for $d_{i}=0$ ] such that the magnetic dissipation term $\mathbf{D}_{J}$ has no contribution to the energy flux through the boundary.

Following Gray et al. (2010), the system is initialized with two initially identical stationary co-axial Bessel-function (also known as Chandrasekhar-Kendall model) spheromaks (Chandrasekhar and Kendall, 1957; Bellan, 2002) with Bfield satisfying

$\nabla \times \mathbf{B}=\lambda \mathbf{B}$

with constant $\lambda$. The spheromaks are placed next to each other with their rotational symmetry axes along the $z$-axis to fill the cylindrical domain such that $\hat{n} \cdot \mathbf{B}=0$ everywhere at the boundary. The $z=1.5 r_{0}$ surface forms an interface between the two spheromaks, such that radial components of the respective magnetic fields are both positive at the interface. The field components along the spheromaks' magnetic axes (referred to as toroidal components) are counterdirected with respect to each other and zero at the interface. A single interior magnetic null point $(|\mathbf{B}|=0)$ is formed at the center of the domain $(r, z)=\left(0,1.5 r_{0}\right)$. Two other

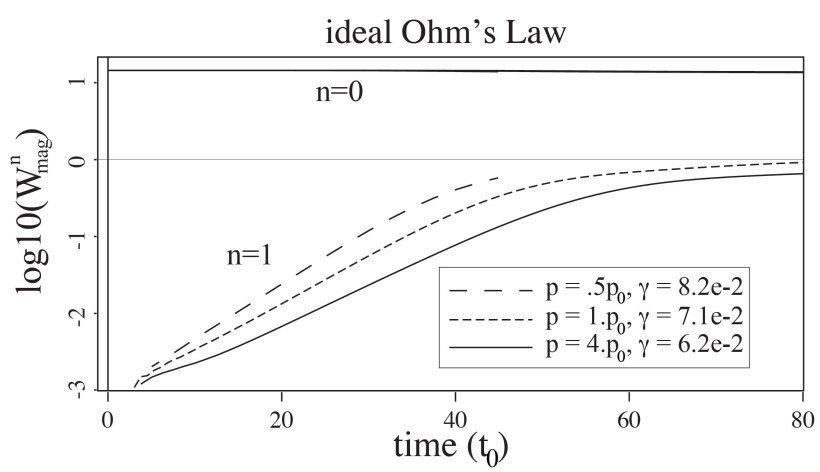

Fig. 2. Time-traces of normalized magnetic energy $W_{\text {mag }}^{n}$ in $n=0$ and $n=1$ modes for three simulations with different values of initial pressure $\left.p\right|_{t=0}=0.5 p_{0}, 1 p_{0}, 4 p_{0}$ all conducted with no magnetic dissipation $\left(\mathbf{D}_{J}=\mathbf{0}\right)$ in the single-fluid regime $\left(d_{i}=0\right)$. The corresponding normalized linear growth rate $\gamma$ of the tilt mode converting $W_{\text {mag }}^{0}$ energy into $W_{\text {mag }}^{1}$ energy is shown in the legend for each of the three cases.

isolated magnetic nulls are initially located on the cylinder end-walls, at $(r, z)=(0,0)$ and $(r, z)=(0, L)$; and three null lines are located on the walls at $(r, \phi, z)=\left(r_{0}, \phi, 0\right)$, $(r, \phi, z)=\left(r_{0}, \phi, 1.5 r_{0}\right)$, and $(r, \phi, z)=\left(r_{0}, \phi, L\right)$. In order to generate the initial numerical representation of $\mathbf{A}$ satisfying these conditions on $\nabla \times \mathbf{A}$, the HiFi framework is utilized to solve a simple differential vector equation of the form:

$\nabla^{2} \mathbf{A}=-\mathbf{J}_{0}=-\lambda \mathbf{B}_{0}$

subject to the boundary conditions $\hat{n} \times \mathbf{A}=\mathbf{0}$ and $\nabla \cdot \mathbf{A}=0$, where

$\mathbf{B}_{0}(r, z) / r_{0} B_{0}=$

$-k_{z} J_{1}(\alpha r) \cos \left(k_{z} z\right) \hat{r}+\lambda J_{1}(\alpha r) \sin \left(k_{z} z\right) \hat{\phi}+\alpha J_{0}(\alpha r) \sin \left(k_{z} z\right) \hat{z}$,

$\lambda=\sqrt{\alpha^{2}+k_{z}^{2}}, k_{z}=2 \pi / L$, and $\alpha r_{0}=3.8317$ is the first zero of the $J_{1}$ Bessel function. The initial plasma density $\rho$ and 

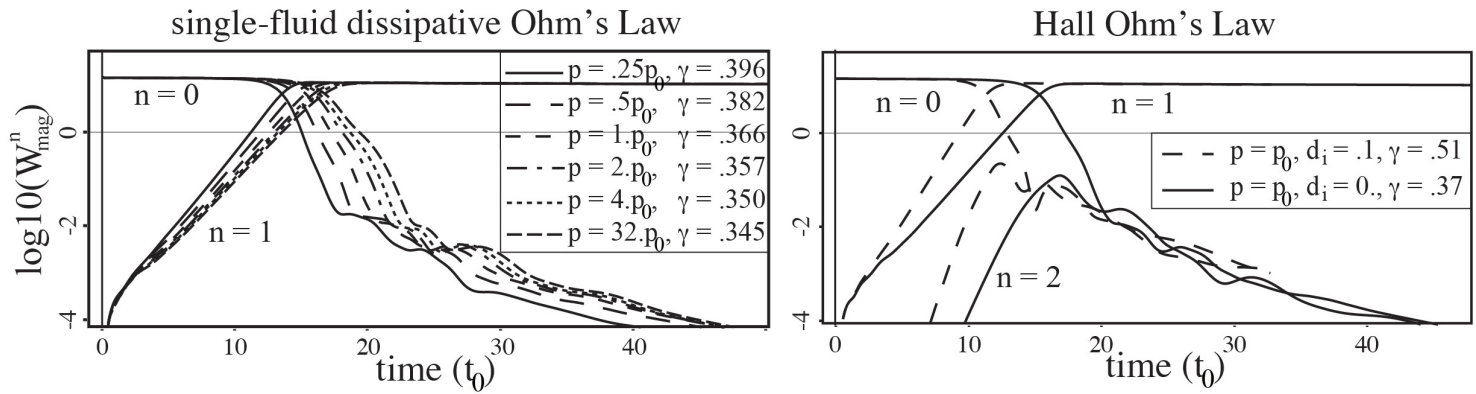

Fig. 3. Comparison of time-traces of $\phi$-mode magnetic energy $W_{\text {mag }}^{n}$ for several simulations (left) with different values of initial pressure $\left.p\right|_{t=0}$ conducted in the single-fluid mode with finite magnetic dissipation $\left(d_{i}=0, v=5 \times 10^{-6}\right)$, and (right) both with and without the Hall effect for the same value of initial plasma pressure and finite magnetic dissipation $\left(d_{i}^{2} \mu_{e}=v=5 \times 10^{-6}\right)$. The corresponding normalized linear growth rate $\gamma$ of the $n=1$ mode is shown in the legend for each of the simulations.

pressure $p$ within the computational domain are taken to be uniform, thus producing a force-free initial equilibrium for the simulations.

\section{Global evolution of the two-spheromak system}

The initial condition described above is an equilibrium with free magnetic energy that can be unstable to aligned tilting of the spheromaks (Gray et al., 2010), shown in its early nonlinear stage in Fig. 1. In the simulations, a tilting perturbation $\hat{z} \cdot \tilde{\rho} \tilde{\mathbf{v}}_{i}=10^{-2}\left(r / r_{0}\right) \cos (\phi) \sin \left(k_{z} z / 2\right)$ is applied to the equilibrium at $t=0$ to break the axisymmetry and initialize the instability. This tilt instability is an ideal MHD instability that converts magnetic energy from the axisymmetric $n=0$ mode to the $n=1$ mode and does not require magnetic dissipation to become unstable (Finn et al., 1981; Bondeson et al., 1981). Below, we investigate the linear and non-linear dynamics of this system using three sets of MHD simulations with qualitatively different Ohm's Law, Eq. (3): ideal MHD $\left(d_{i}=\mu_{e}=0, \mathbf{D}_{J}=\mathbf{0}\right)$, dissipative single-fluid MHD $\left(d_{i}=\mu_{e}=0, \mathbf{D}_{J} \neq \mathbf{0}\right)$, and dissipative Hall MHD $\left(d_{i} \neq 0\right.$, $\left.\mathbf{D}_{J} \neq \mathbf{0}\right)$.

Figure 2 shows time-traces of normalized magnetic energy $W_{\mathrm{mag}}^{n}$ in both $n=0$ and $n=1$ modes from three simulation runs with the ideal MHD Ohm's Law and three different initial plasma temperature values, where the $n$ th-mode magnetic energy is calculated as

$$
W_{\mathrm{mag}}^{n} \equiv \frac{a_{n}}{r_{0}^{3} B_{0}^{2}} \sum_{j=1}^{3} \int_{0}^{r_{0}} \int_{0}^{L}\left|\int_{0}^{2 \pi} B_{j} \exp (i n \phi) d \phi\right|^{2} d z r d r,
$$

with $a_{0}=0.5$ and $a_{n>0}=1$. These three, as well as all other simulations described below have been conducted with $\left.\left(\rho / \rho_{0}\right)\right|_{t=0}=1$ and normalized heat conduction and ion viscosity coefficients of $\kappa=\mu_{i}=10^{-2}$. It is apparent from the figure that the $n=1$ tilt mode is indeed unstable with linear growth rate $\gamma \equiv 0.5\left\{\partial\left[\ln \left(W_{\mathrm{mag}}^{n}\right)\right] / \partial \tilde{t}\right\}$ weakly sensitive to the background plasma pressure. However, it is also clear that in

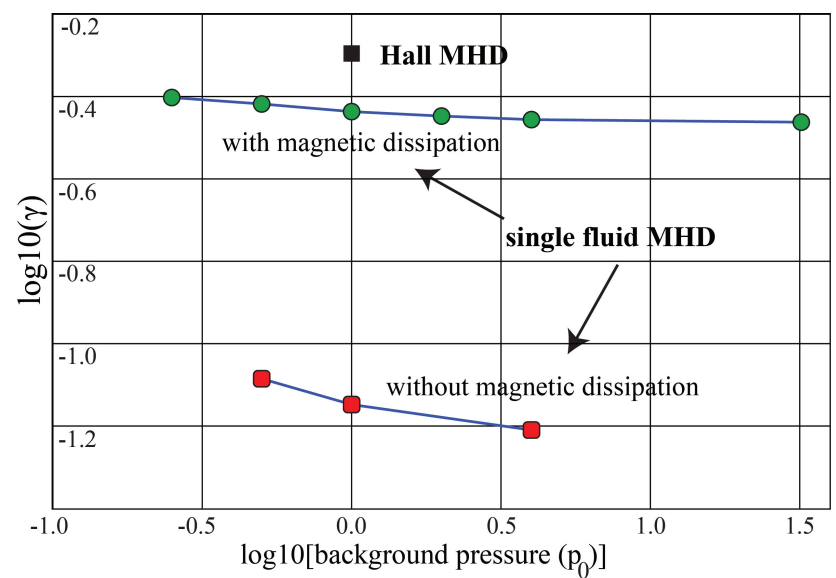

Fig. 4. Growth rates of $n=1$ tilt perturbation in the magnetic field as a function of background pressure from the simulations shown in Figs. 2-3.

the absence of magnetic dissipation the tilt mode saturates at small amplitude as no topological changes in magnetic connectivity can take place either within or across the two initial spheromak flux bundles.

The situation becomes dramatically different in the presence of magnetic dissipation, when magnetic reconnection between the two topologically separated flux volumes is allowed to take place. With finite magnetic dissipation, as the spheromaks begin to tilt, the $\mathbf{B}$-field at the magnetic null is stressed and magnetic reconnection at the central magnetic null between the "bottom" of the left spheromak and "top" of the right spheromak is initiated, as shown in the left panel of Fig. 1. Time-traces of $W_{\mathrm{mag}}^{n}$ from a series of simulations with non-zero magnetic dissipation are shown in Fig. 3. The timetraces show that unlike the ideal Ohm's Law cases, in the simulations with non-zero $\mathbf{D}_{J}$ nearly all of the initial axisymmetric magnetic energy $W_{\text {mag }}^{0}$ is transferred into the helical mode energy $W_{\text {mag }}^{1}$, less the free magnetic energy released during the reconnection and relaxation process. 


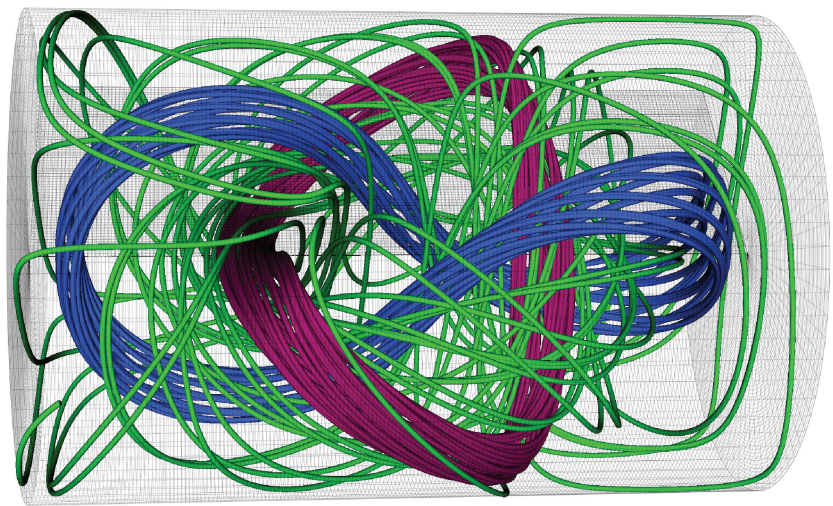

Fig. 5. Three magnetic field lines of the saturated minimum magnetic energy $n=1$ helical state (Cothran et al., 2010) from the single-fluid HiFi computation with $\left.p\right|_{t=0}=1 p_{0}$ and $v=5 \times 10^{-6}$ in the $L: r_{0}=3: 1$ computational domain used in this paper.

The left panel of Fig. 3 shows results from six single-fluid simulations with $v=5 \times 10^{-6}$ and six different initial plasma pressures, from $\left.p\right|_{t=0}=0.25 p_{0}$ up to $\left.p\right|_{t=0}=32 p_{0}$; the tilt growth rate $\gamma$ for each of the simulations is shown in the legend. In agreement with the zero-dissipation results, the higher $\beta$ runs again show systematically lower growth rates. But the presence of magnetic dissipation also increases $\gamma$ by nearly an order of magnitude. It should be noted that the stability properties of this two-spheromak system to the coaligned tilt mode are very similar to those of the internal kink mode, which is a helical ideal MHD instability extensively studied in tokamaks and other toroidally confined plasmas (Rosenbluth et al., 1973). It is well known that the ideal internal kink instability growth rate is also greatly enhanced by the presence of magnetic dissipation and field-line tearing (Coppi et al., 1976; Lukin, 2008), and even further accelerated in the presence of two-fluid effects (Porcelli, 1991).

Introducing two-fluid effects into the present numerical experiment, two simulations, one with and one without the Hall effect, are compared in the right panel of Fig. 3. The two cases have been set up to have approximately the same form and magnitude of magnetic dissipation by choosing $d_{i}^{2} \mu_{e}=$ $5 \times 10^{-6}$ in the Hall MHD simulation and $v=5 \times 10^{-6}$ in the single-fluid MHD simulation. (Note that magnetic dissipation in simulations with $d_{i} \neq 0$ is due to the term $\left(-d_{i}^{2} \mu_{e} / \rho\right) \nabla^{2}(\mathbf{J} / \rho)$ within $\mathbf{D}_{J}$ in Eq. (3), and $\rho / \rho_{0} \approx 1$ has been assumed.) There are two separate but related physical effects that can be expected to influence the linear evolution of the tilt mode for $d_{i}>0$. On the global scale, ion inertia can serve to allow the magnetic field structure to begin to tilt ahead of the ion fluid, thereby allowing for faster acceleration and instability growth rate. This is an ideal Hall MHD effect that can be expected to become significant for $d_{i} \approx O(1)$ and does not allow for any change in magnetic topology. However, on small scales, it is well known that whenever $d_{i}>0$, magnetic dissipation is sufficiently small but non-negligible, and there are regions of stressed sheared magnetic field unstable to tearing, finite ion inertia allows magnetic field to slip through the ion fluid and reconnect at rates substantially faster than those for $d_{i}=0$, with everything else being equal (Drake and Lee, 1977; Terasawa, 1983). In the semicollisional regime with $d_{i}=0.1$ and $\left(p_{e} / p_{i}\right)=1$ chosen for this study, it is the latter mechanism that is likely the dominant factor in increasing the linear tilt growth rate by $\approx 38 \%$.

The measured $n=1$ tilt growth rates from simulations with the ideal, dissipative single-fluid and dissipative Hall MHD subsets of PDEs are collected together and plotted on a log-log plot versus background plasma pressure in Fig. 4 . As discussed above, it is clear that while variation in plasma $\beta$ from $\beta<1$ to $\beta \gg 1$ has a systematic effect on the tilt growth rate, it is much weaker than the increase in the growth rate due to non-ideal MHD effects that allow magnetic tearing and reconnection between the two topologically separate spheromak flux bundles to take place. From here onward we will focus only on the simulations with non-zero magnetic dissipation where nearly all of the magnetic energy initially contained in the axisymmetric $n=0$ fields is transferred into the helical $n=1$ mode that constitutes the minimum magnetic energy state in this system (Woltjer, 1958; Taylor, 1974; Cothran et al., 2010; Gray et al., 2010). It should be noted that the final helical state appears to be insensitive to plasma $\beta$ and to the ion inertial effects in the domain geometry and for the plasma parameters investigated here. Figure 5 illustrates the magnetic field lines of this $n=1$ state from one of the single-fluid calculations.

\section{Correlated dynamics of the magnetic null and the 3-D reconnection region}

A schematic of the reconnection region within the global magnetic field structure during early evolution of the system is sketched out in the left panel of Fig. 1, where the components of the $\mathbf{B}$-field in the reconnection plane and of the out-of-plane field being convected into the RR are shown. Here, by the reconnection plane we mean the plane passing through the magnetic null and normal to the rotation axis of the tilt. Figure 6 shows a more detailed representation of the spine-fan magnetic null (Priest and Titov, 1996; Priest and Pontin, 2009) where magnetic reconnection is initiated. The figure illustrates how the location of the magnetic null at each subsequent time-instance is determined by the RR inflows and magnetic field configuration in the immediate neighborhood of the null: As magnetic reconnection commences, the symmetry of the null is broken and reconnection inflows begin to convect inward the components of magnetic field perpendicular to the plane defined by the reconnecting field at the null itself. These field components, originally the toroidal fields of the spheromaks $\hat{\phi} \cdot \mathbf{B}_{0}$, are co-aligned and combine with the magnetic field fan around the null. As a 


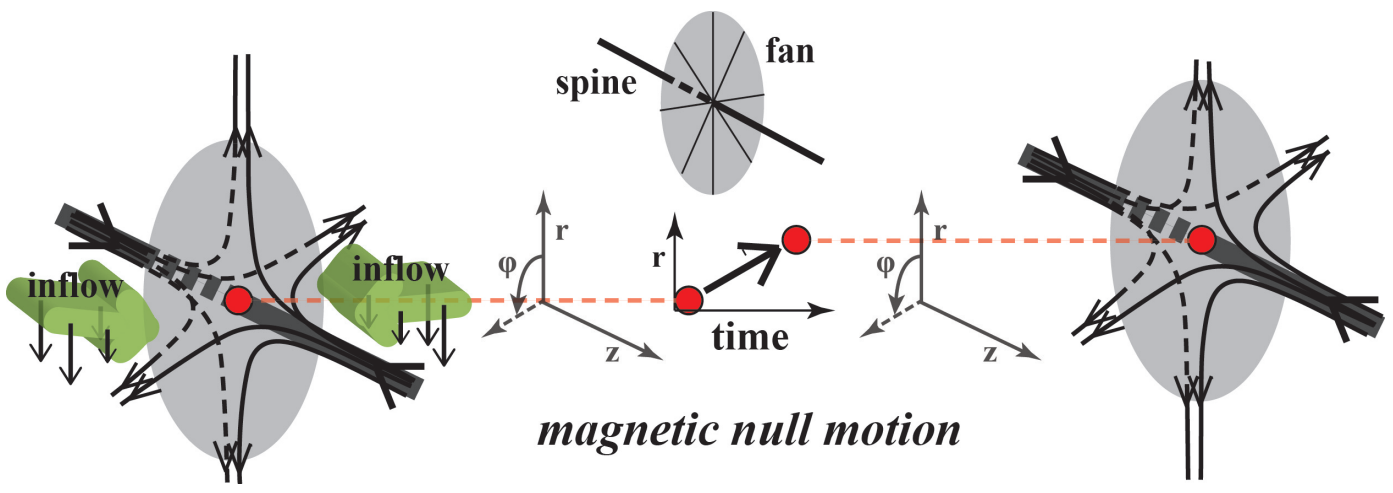

Fig. 6. Schematic of the spine-fan magnetic null centered between the two spheromaks. As the symmetry of the null is broken and magnetic reconnection commences, in-plane plasma flows carry into the null the component of magnetic field perpendicular to the reconnection plane. These field components, originally the toroidal fields of the spheromaks, are co-aligned and combine with corresponding components of the fan magnetic field around the null. As a result, the magnetic null moves along a radial cord in the plane of the fan and normal to the reconnection plane.

result, the magnetic null moves along a radial cord in the plane of the fan and normal to the reconnection plane.

Simulation snapshots of the coincident null and 3-D RR between the two flux bundles from a single-fluid low $\beta$ run with $\left.p\right|_{t=0}=0.25 p_{0}$ and $v=5 \times 10^{-6}$ are shown at $t=12.61 t_{0}$ in Fig. 7. The view of panel (a) supports the schematic of Fig. 6 and demonstrates that as the spine-fan magnetic null is sheared, enhanced values of reconnectionassociated $E_{\mathrm{par}} \equiv \mathbf{E} \cdot \mathbf{B} /|\mathbf{B}|$ (Schindler et al., 1988; Hesse and Schindler, 1988) and current density are observed in the immediate neighborhood of the null, as the null itself is displaced from the original rotational symmetry axis of the spheromaks at $r=0$. Figure 7b shows the same 3-D image viewed from the top with respect to the view of Fig. 7a and such that the reconnection plane is in the plane of the figure. It is apparent that the classic 2-D description of antiparallel reconnection with a thin elongated reconnection current sheet (Sweet, 1958; Parker, 1957) is a reasonable description of the process observed here in the plane containing the magnetic null and in the frame of reference of the RR itself. However, it is also clear that the actual 3-D topology of the magnetic field lines around the null is substantially more complicated with order unity ratio of out-of-reconnectionplane to in-reconnection-plane B-field components immediately away from the null.

It is desirable to unambiguously evaluate the rate of magnetic reconnection $R_{\text {rec }}$ through a 3-D RR. General wellposed prescriptions for doing so in finite-B reconnection or in the presence of magnetic null lines exist in the literature and rely on the value of the integral of $E_{\mathrm{par}}$ along magnetic field lines or on the value of $\mathbf{E}$ along the magnetic separator, respectively (Hesse and Schindler, 1988). While we are not aware of such a prescription for a generic dynamically changing and moving RR in the presence of an isolated magnetic null, a way to calculate the reconnection rate through a sheared magnetic null that relies on certain symmetry of the magnetic fan surface has been previously introduced by Pontin et al. (2005) (see Figure 6 of Pontin et al. (2005)). As applied to the magnetic configuration considered here, this prescription is sufficient and requires a path integral $R_{\text {rec }} \equiv \int_{P_{\text {rec }}} \tilde{\mathbf{E}} \cdot \mathbf{d} \tilde{x}$ along the path $P_{\text {rec }}$ forming a straight line through the magnetic null and parallel to the tilt rotation axis of the two-spheromak system. In fact, that is the same line as the one traversed by the magnetic null and corresponds to the vertical line through the null in the magnetic fan plane of Fig. 6 and to the $\hat{x}$-directed line through the magnetic null in Fig. 7, such that $\tilde{\mathbf{B}} \times \mathbf{d} \tilde{P}_{\text {rec }}=\mathbf{0}$ everywhere along $P_{\text {rec }}$ and $\int_{P_{\mathrm{rec}}} \tilde{\mathbf{E}} \cdot \mathbf{d} \tilde{x}=\int\left|\tilde{E}_{\mathrm{par}}\right| d \tilde{P}_{\mathrm{rec}}$.

Figure 8 a shows time traces of $\log _{10}\left[R_{\text {rec }}\right]$ from the simulations already discussed in Sect. 3: six dissipative singlefluid MHD simulations with different $\left.p\right|_{t=0}$, labeled A-F, and a Hall MHD simulation with $d_{i}=0.1$ and $\left.p\right|_{t=0}=1 . p_{0}$, labeled $\mathbf{H}$. In all cases, $R_{\text {rec }}$ is observed first to grow exponentially from negligible values early in the simulations, second to grow super-exponentially and increase its effective growth rate (the slope of the curves in Fig. 8a), and third to peak and rapidly decay after another secondary peak. The effective growth rates in the early linear and later nonlinear phases of reconnection $\gamma_{R} \equiv\left\{\partial\left[\ln \left(R_{\text {rec }}\right)\right] / \partial \tilde{t}\right\}$ are shown versus $\left.p\right|_{t=0}$ on a log-log plot in Fig. 8 b.

A comparison of the early linear growth rates $\gamma_{R}$ to the corresponding tilt mode growth rates shown in Fig. 4 demonstrates a good quantitative agreement between the two sets of values. This is consistent with the interpretation of the observed growth rates as those for a tearing-accelerated coaligned linear tilt mode given in Sect. 3. The observed super-exponential increase in $R_{\text {rec }}$ during the transition to the nonlinear reconnection phase followed by saturation and rapid decay is also a familiar phenomenon previously observed in strongly unstable 2-D single-fluid resistive tearing 


\section{(a)}
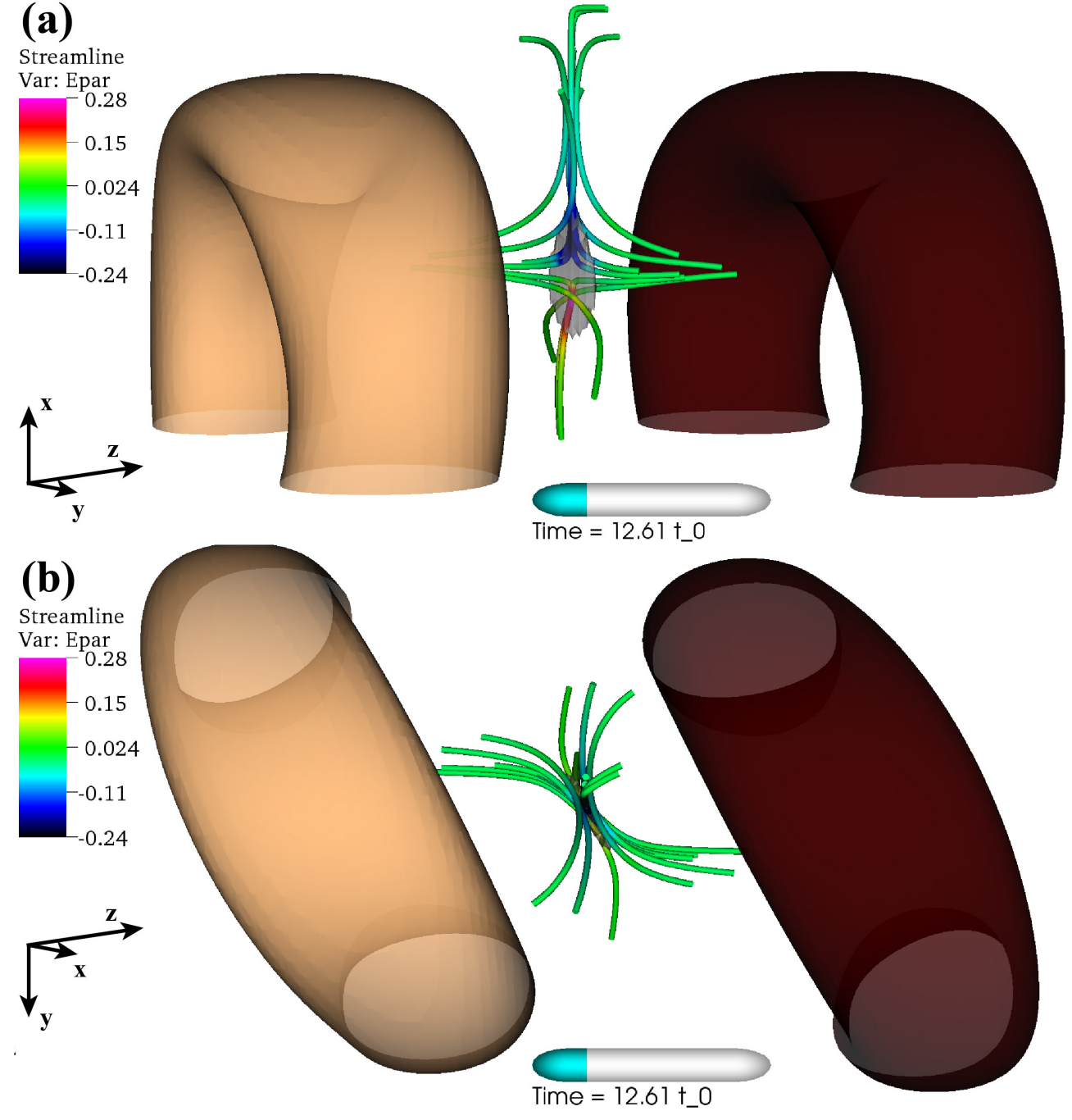

Fig. 7. Snapshots of the 3-D RR between the two flux bundles from a simulation with $\left.p\right|_{t=0}=0.25 p_{0}, d_{i}=0$, and $v=5 \times 10^{-6}$ at $t=12.61 t_{0}$. A view of the spine-fan magnetic null similar to that of Fig. 6 is shown in panel (a) and a view from the top is shown in panel (b). Light and dark brown surfaces are those of constant $r \hat{\phi} \cdot \mathbf{A}$ and approximate magnetic flux surfaces, the streamlines around the null are select magnetic field lines with the color showing parallel $\mathbf{E}$-field $\tilde{E}_{\text {par }}=\tilde{\mathbf{E}} \cdot \mathbf{B} /|\mathbf{B}|$ at each location along the field lines, and a gray surface in the center of the null is that of enhanced current density $|\tilde{\mathbf{J}}|=30$. associated with ongoing magnetic reconnection.

mode reconnection (Loureiro et al., 2005) and nonlinear $m=1$ internal kink mode simulations that included twofluid effects (Aydemir, 1992; Lukin, 2008). This explosive behavior of a reconnecting system is commonly explained by the self-driven nature of nonlinear magnetic reconnection (Drake, 2001; Lukin, 2008).

Another quantity that is simple to evaluate and necessarily has a strong correlation with the reconnection rate is the magnitude of normalized electric field due to magnetic dissipation:

$E_{\mathrm{diss}} \equiv\left\{\begin{array}{r}\frac{d_{i}^{2} \mu_{e}}{\tilde{\rho}} \mid \begin{array}{l}\tilde{\nabla}^{2}(\tilde{\mathbf{J}}) \mid, \quad d_{i}>0 \\ v\end{array} \\ v\left|\tilde{\nabla}^{2} \tilde{\mathbf{J}}\right|, \quad d_{i}=0\end{array}\right.$.
Since magnetic reconnection requires magnetic dissipation, $E_{\text {diss }} \ll 1$ everywhere outside of the RR, and $E_{\text {diss }} \geq\left|\tilde{E}_{\text {par }}\right|$ everywhere on $P_{\mathrm{rec}}$, it is reasonable to associate the physical location of maximum $E_{\text {diss }}$ within the domain with the location where the most intense magnetic reconnection takes place. In fact, throughout the reconnection process, the location of $\max \left(E_{\text {diss }}\right)$ over the domain remains somewhere on $P_{\text {rec }}$.

Figure 9 a shows the time evolution of $\max \left(E_{\text {diss }}\right)$ during the period of most intense reconnection for the six dissipative single-fluid MHD simulations. The corresponding locations of the magnetic null and the point of maximum $E_{\text {diss }}$ along the radial cord traveled by both, $r_{\text {null }}$ and $r_{\mathrm{RR}}$ respectively, are shown in Fig. 9b, c versus time. Figure 9d shows the 

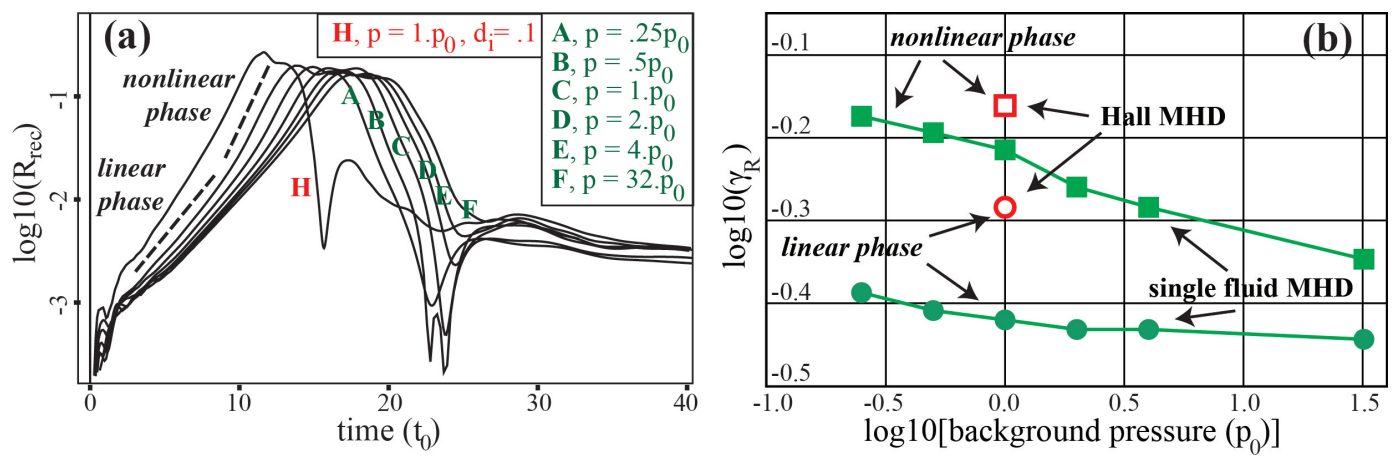

Fig. 8. (a) Time traces of normalized reconnection rate $R_{\mathrm{rec}}$ for the six single-fluid MHD simulations with varying plasma $\beta$ (labeled A-F) and the Hall MHD simulation (labeled $\mathbf{H}$ ) described in Fig. 3. (b) The effective growth rate $\gamma_{R}$ of $R_{\text {rec }}$ versus the background pressure in the early linear and late nonlinear phases of reconnection.
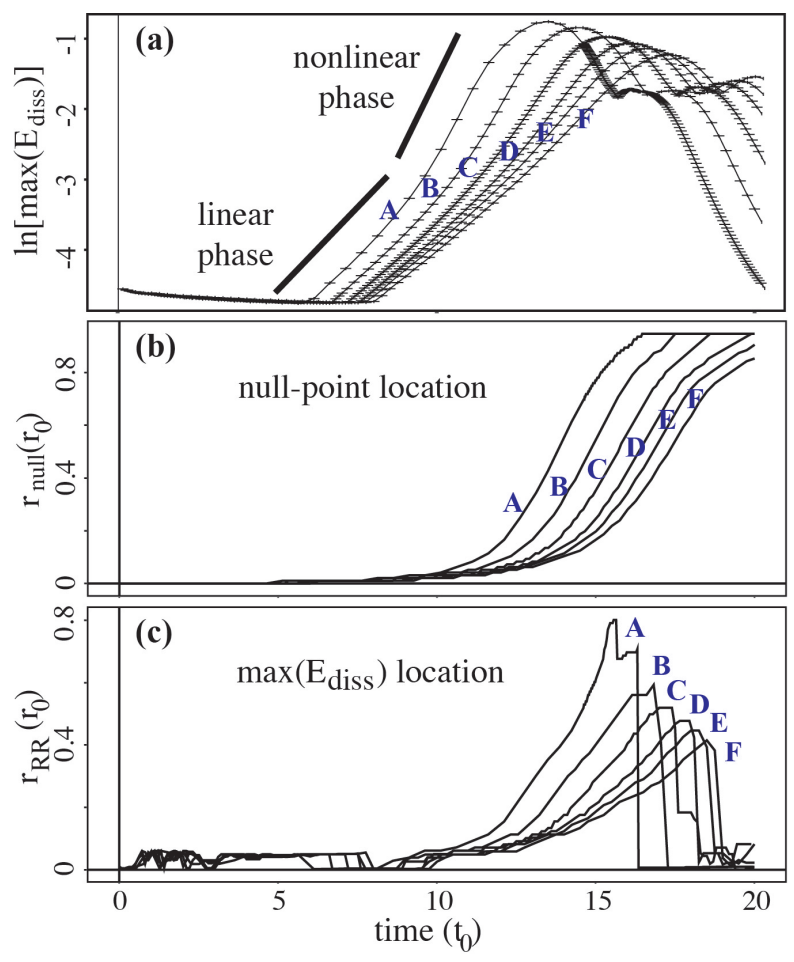

$\mathbf{A}, \mathrm{p}=0.25 \mathrm{p}_{0}$
$\mathbf{B}, \mathrm{p}=0.5 \mathrm{p}_{0}$
$\mathbf{C}, \mathrm{p}=1.0 \mathrm{p}_{0}$
$\mathbf{D}, \mathrm{p}=2.0 \mathrm{p}_{0}$
$\mathbf{E}, \mathrm{p}=4.0 \mathrm{p}_{0}$
$\mathbf{F}, \mathrm{p}=32.0 \mathrm{p}_{0}$

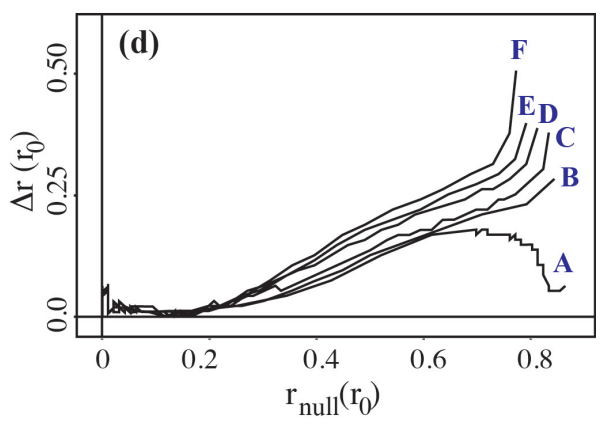

Fig. 9. Time traces of (a) the maximum magnitude of normalized dissipation electric field $\max \left(E_{\mathrm{diss}}\right)$; (b) the radial position of the magnetic null-point $r_{\text {null }}$ and (c) the radial position of the location of maximum dissipation E-field $r_{\mathrm{RR}}$ for the six single-fluid simulations with varying plasma $\beta$. Panel (d) shows the radial distance $\Delta r \equiv\left(r_{\text {null }}-r_{\mathrm{RR}}\right)$ versus the null-point's radial position $r_{\text {null }}$.

distance between the two, $\Delta r \equiv\left(r_{\text {null }}-r_{\mathrm{RR}}\right)$, as a function of $r_{\text {null }}$ while the null is located within the simulation domain. There are several observations that can be made by examining Figs. 8-9 in concert: (1) the magnitudes and the growth rates of $R_{\text {rec }}$ and $\max \left(E_{\mathrm{diss}}\right)$ are greatest while the the magnetic null and the RR are moving and co-located; (2) simulations with lower $\beta$ show both stronger spatial correlation between the locations of the null and the RR and higher magnitudes of $R_{\text {rec }}$ and $\max \left(E_{\text {diss }}\right)$; (3) simulations with lower $\beta$ also show stronger super-exponential growth of
$R_{\text {rec }}$ indicated by the difference between the nonlinear and linear growth rates in Fig. 8b. All these point towards a relationship between the observed explosive growth of the reconnection rate and the location of the RR relative to the moving magnetic null.

The speed of the moving RR in the single-fluid simulations discussed above can be computed from Fig. $9 \mathrm{c}$ in a straightforward manner and can achieve up to $0.24 v_{\mathrm{Alf}}$ for the lowest $\beta$ simulations shown. However, the apparent motion of the $\mathrm{RR}$ and the null is not associated with any plasma flow in the 


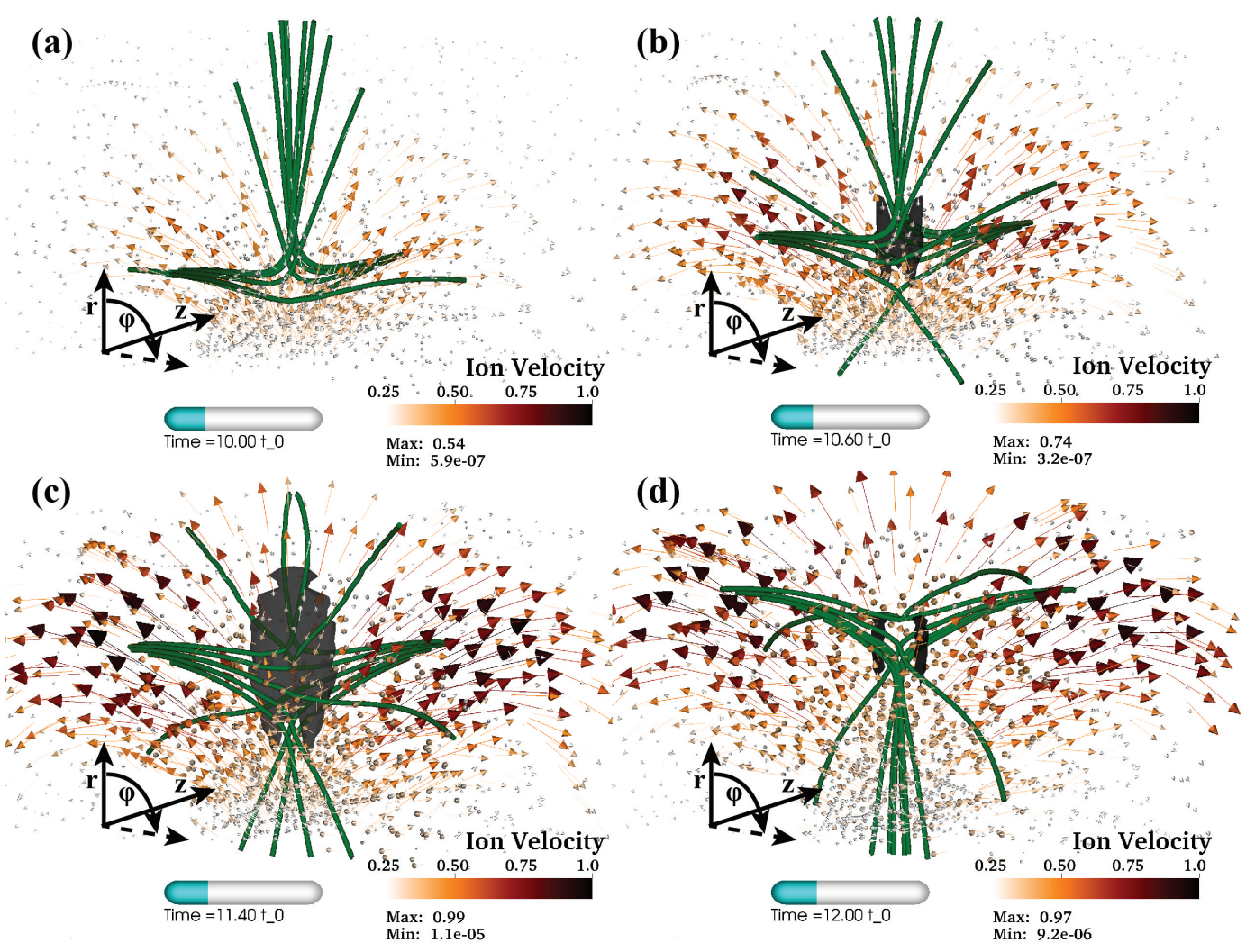

Fig. 10. Four snapshots of the 3-D RR from a Hall MHD simulation with $\left.p\right|_{t=0}=p_{0}, d_{i}=0.1$, and $d_{i}^{2} \mu_{e}=5 \times 10^{-6}$ before [panel (a)], during [panels (b)-(c)] and after [panel (d)] onset of fast reconnection through the moving magnetic null. In each panel, the streamlines denote magnetic field lines traced from a set of five pairs of initial points located at $r=\{0.1,0.2,0.3,0.4,0.5\} r_{0}$ along the radial cord traveled by the null, with each origin pair separated by $0.02 r_{0}$. The arrows denote the ion flow field $\tilde{\mathbf{v}}_{i}$ with both the color and the size of the arrows indicating the magnitude of the flow, and the gray surfaces in panels (b)-(d) denote enhanced current density $|\tilde{\mathbf{J}}|=32$. associated with ongoing magnetic reconnection. The orientation and viewpoint of the RR is the same in all panels and similar to that shown in Fig. 6 and Fig. 7 a above.

direction of this motion. Instead, the plasma flow accelerated by the released tension of reconnected field lines stays parallel to the reconnection plane, as the reconnection plane moves with the RR in the direction normal to itself (see Fig. 8 of Gray et al., 2010). This situation becomes substantially different when the ion inertial effects are taken into account by setting $d_{i}>0$ and sufficiently large to be greater than the magnetic dissipation scale within the RR.

Figure 10 shows the evolution of magnetic field and ion flow around the radial chord traveled by the magnetic null and the RR during reconnection in the Hall MHD regime with $d_{i}=0.1$ and $d_{i}^{2} \mu_{e}=5 \times 10^{-6}$. It is clear that in this case the plasma flow pattern in the immediate neighborhood of the $\mathrm{RR}$ is fully three-dimensional with a substantial component of the flow directed along the upward motion of the RR itself. Both the magnitude and direction of this vertical ion flow in the RR, which is also co-aligned with the direction of RR current density, is consistent with the strong currentaligned ion flows previously observed in 3-D Hall MHD sim- ulations of localized reconnection within an infinitely long current channel by Huba and Rudakov (2002). These ion flows carry some of the reconnection-associated plasma current, but since the peak value of the vertical component of $\mathbf{v}_{i}$ in Fig. 10 is $\approx 0.5 v_{\text {Alf }}$ and $d_{i}|\tilde{\mathbf{J}}|>3.0$, most of the reconnection current in this simulation is carried by the electrons.

As noted is Sect. 1, previous simulations of a 3-D RR in the two-fluid and kinetic regimes, including those by Huba and Rudakov (2002), have shown that the location of reconnection onset always propagates in the direction of motion of the dominant current carriers (Lapenta et al., 2006). The example presented here clearly contradicts that notion, as the RR and the null shown in Fig. 10 are moving upward, while the current-carrying electron flow is directed downward. The explanation for this apparent contradiction appears to be straightforward: The previous work considered equilibria which were globally 2-D with a small localized 3$\mathrm{D}$ perturbation, leaving the direction of the RR propagation to be determined by the difference in the ion and electron 
current-carrying flow. By contrast, in the globally 3-D configuration considered here, it is the propagation of the 3-D minimum in the magnetic pressure associated with the magnetic null that is the determining factor for the direction of the RR propagation. This mechanism operates equally well both in the single-fluid and Hall MHD regimes, and thus the ion inertial effects do not qualitatively change the dynamics of reconnection through this moving 3-D magnetic null.

\section{Conclusions}

The numerical simulations of null-point reconnection between two spheromak flux-ropes described above provide a novel view of 3-D magnetic reconnection by highlighting the importance of considering globally three-dimensional magnetic configurations which are free to evolve and undergo magnetic relaxation without being constrained by nearby line-tying or other similarly restrictive boundary conditions.

A state-of-the-art implicit spectral element multi-fluid modeling framework HiFi has been used to conduct the single-fluid and Hall MHD simulations to investigate both the relationship between the global magnetic structure and the RR evolution, and some of the details of the RR dynamics. It should be noted that one important measure of the accuracy of the HiFi modeling framework is the fact that in the absence of magnetic dissipation being explicitly included in the Ohm's Law [Eq. (3)], no magnetic reconnection is observed in the simulations, as demonstrated by comparing Figs. 2 and 3. This valuable feature of the HiFi framework is due to the use of both spectral element spatial discretization, where all spatial derivatives are evaluated analytically, and the non-dissipative implicit Crank-Nicolson time-advance.

The effect of the small-scale magnetic dissipation on the global dynamics of the two-spheromak system, and in particular on the linear tilting instability that sets off the non-linear reconnection phase, is documented in Figs. 2-4. It is shown that the magnetic tearing enabled by the magnetic dissipation can dramatically increase the tilt growth rate even in high- $\beta$ plasmas, and the ion inertial effects can further accelerate the development of the mode. And what is even more important, the magnetic dissipation allows for the nonlinear topological change in the global magnetic field configuration and relaxation to the lowest magnetic energy $n=1$-dominated state shown in Fig. 5.

Throughout the manuscript, a special emphasis has been made on the importance of the observed correlation between the motion of the magnetic null and of the RR region. There are two primary reasons why we believe that this effect is an important one:

1. The tendency of the RR to follow the motion of the magnetic null, particularly in low- $\beta$ plasmas and even in the absence of any two-fluid or kinetic effects, could go a long way towards explaining the observed motion and variability of the emission signatures of magnetic reconnection (Qiu, 2009) in such topologically complex magnetic system as the solar corona. In future work, we intend to look for a similar effect in magnetic configurations with localized magnetic pressure minima, but non-vanishing $|\mathbf{B}|$ everywhere.

2. The possibility of nearly Alfvénic motion of the reconnection region through the global magnetic field structure implies that the location of plasma acceleration due to magnetic reconnection can also move through the structure; i.e. if at $t_{0}$ plasma is being accelerated at some location $\mathbf{x}_{0}$, then at some later time $t_{1}$ plasma is no longer accelerated at $\mathbf{x}_{0}$ but is at some other location $\mathbf{x}_{1}$. Whenever that is the case, many of the usual 2-D reconnection paradigms, such as the Sweet-Parker outflow bottleneck (Sweet, 1958; Parker, 1957), may become irrelevant since the time it takes plasma to flow through a RR becomes comparable to the displacement time of the RR itself.

The following simple steady-state analysis illustrates the second point. Assume a 3-D localized RR of width $\delta_{R R}$ determined by the non-ideal magnetic dissipation processes across the RR, length $L_{\mathrm{RR}}$ along the reconnection outflow direction, and some height $H_{\mathrm{RR}}$ along the direction of reconnection current. Also, assume that the magnetic field configuration of the RR is moving at some velocity $\mathbf{v}_{\mathrm{RR}}$ along the direction of the reconnection current, as illustrated in Fig. 6. Then, in steady state, in the moving frame of reference of the RR,

$$
\begin{aligned}
\frac{d L_{\mathrm{RR}}}{d t} & =\frac{\partial L_{\mathrm{RR}}}{\partial \tilde{t}}+\mathbf{v}_{\mathrm{RR}} \cdot \nabla L_{\mathrm{RR}}=0 \\
& \Rightarrow L_{\mathrm{RR}} \approx \frac{v_{e l}}{v_{\mathrm{RR}}} \frac{H_{\mathrm{RR}}}{2},
\end{aligned}
$$

where $v_{e l} \equiv\left(\partial L_{\mathrm{RR}} / \partial t\right)$ is the rate of nonlinear elongation of a 2-D reconnection current layer in the stationary frame of reference. It has been previously shown in 2-D incompressible single-fluid simulations that $v_{e l}$ can be a large fraction, up to a half, of the upstream Alfvén velocity $v_{\text {Alf }}$ (Lukin, 2008). The 3-D results reported above show that $v_{\mathrm{RR}}$ can also achieve a large fraction of $v_{\text {Alf }}$, up to $v_{\mathrm{RR}}=0.24 v_{\mathrm{Alf}}$ in the smallest $\beta$ single-fluid simulations attempted. Thus, it follows that $L_{\mathrm{RR}} / H_{\mathrm{RR}}$ can be of order unity, which is consistent with the RR current density structures shown in Fig. 7 and Fig. 10.

While in the Sweet-Parker model $L_{\mathrm{RR}}$ is assumed to be the global scale of the reconnecting magnetic structures, which leads to extremely slow reconnection in astrophysical-size systems; the question now becomes: what determines $H_{\mathrm{RR}}$ and how small can that be? The evidence presented here points towards some mesoscale determined by local variations in $|\mathbf{B}|$ of the reconnecting magnetic structures that is much greater than the dissipation scale, but also much smaller than the global scale. Yet, the simulation results 
discussed above do not provide sufficient separation of scales to affirmatively answer that question, leaving it for future work.

Acknowledgements. We gratefully acknowledge the contributions of A. H. Glasser and W. Lowrie to the development of the HiFi code. We also thank M. R. Brown and T. Gray for helpful discussions and the anonymous referees for many helpful comments. This work has been funded by the US Office of Naval Research and NASA SR\&T and LWS programs. The computing time necessary for the simulation results reported in the manuscript has been granted under the US DOD High Performance Computing Modernization Program.

Edited by: G. Lapenta

Reviewed by: two anonymous referees

\section{References}

Aydemir, A. Y.: Nonlinear studies of $\mathrm{m}=1$ modes in hightemperature plasmas, Phys. Fluids B, 4, 3469-3472, 1992.

Bellan, P. M.: Generalization of cylindrical spheromak solution to finite beta and large reversed shear, Phys. Plasmas, 9, 30503056, 2002.

Bhattacharjee, A., Germaschewski, K., and Ng, C. S.: Current singularities: drivers of impulsive reconnection, Phys. Plasmas, 12, 042305, 2005.

Birn, J., Drake, J. F., Shay, M. A., Rogers, B. N., Denton, R. E., Hesse, M., Kuznetsova, M., Ma, Z. W., Bhattacharjee, A., Otto, A., and Pritchett, P. L.: Geospace Environment Modeling (GEM) magnetic reconnection challenge: Resistive tearing, anisotropic pressure and Hall effects, J. Geophys. Res., 106, 3715-3719, 2001.

Bondeson, A., Marklin, G., An, Z. G., Chen, H. H., Lee, Y. C., and Liu, C. S.: Tilting instability of a cylindrical spheromak, Phys. Fluids, 24, 1682-1688, 1981.

Chandrasekhar, S. and Kendall, P. C.: On Force-Free Magnetic Fields, Astrophys. J., 126, 457-460, 1957.

Coppi, B., Galvão, R., Pellat, R., Rosenbluth, M., and Rutherford, P.: Resistive internal helical modes, Fiz. Plazmy, 2, 961-966, 1976.

Cothran, C. D., Landerman, M., Brown, M. R., and Matthaeus, W. H.: Three-dimensional structure of magnetic reconnection in a laboratory plasma, Geophys. Res. Lett., 30, 1213, doi:10.1029/2002GL016497, 2003.

Cothran, C. D., Brown, M. R., Gray, T., Schaffer, M. J., Marklin, G., and Lukin, V. S.: Observation of a nonaxisymmetric magnetohydrodynamic self-organized state, Phys. Plasmas, 17, 055705, doi:10.1063/1.3327214, 2010.

Daughton, W., Roytershteyn, V., Albright, B. J., Karimabadi, H., Yin, L., and Bowers, K. J.: Transition from collisional to kinetic regimes in large-scale reconnection layers, Phys. Rev. Lett., 103, 065004, doi:10.1103/PhysRevLett.103.065004, 2009.

Drake, J. F.: Magnetic explosions in space, Nature, 410, 525-526, 2001.

Drake, J. F. and Lee, Y. C.: Nonlinear evolution of collisionless and semicollisional tearing modes, Phys. Rev. Lett., 39, 453456, 1977.
Finn, J. M., Manheimer, W. M., and Ott, E.: Spheromak tilting instability in cylindrical geometry, Phys. Fluids, 24, 1336-1341, 1981.

Fletcher, L., Metcalf, T. R., Alexander, D., Brown, D. S., and Ryder, L. A.: Evidence for the flare trigger site and three-dimensional reconnection in multiwavelength observations of a solar flare, Astrophys. J., 554, 451-463, 2001.

Frank, A. G. and Bogdanov, S. Y.: Experimental study of current sheet evolution in magnetic configurations with null-points and X-lines, Earth Planets Space, 53, 531, 2001.

Glasser, A. H. and Tang, X. Z.: The SEL macroscopic modeling code, Comp. Phys. Comm., 164, 237-243, 2004.

Gray, T., Lukin, V. S., Brown, M. R., and Cothran, C. D.: Threedimensional reconnection and relaxation of merging spheromak plasmas, Phys. Plasmas, 17, 102106, doi:10.1063/1.3492726, 2010.

Hesse, M. and Schindler, K.: A Theoretical Foundation of General Magnetic Reconnection, J. Geophys. Res., 93, 5559-5567, 1988.

Huba, J. D. and Rudakov, L. I.: Three-dimensional Hall magnetic reconnection, Phys. Plasmas, 9, 4435-4438, 2002.

Intrator, T. P., Sun, X., Lapenta, G., Dorf, L., and Furno, I.: Experimental onset threshold and magnetic pressure pile-up for 3-D reconnection, Nat. Phys., 5, 521-526, 2009.

Katz, N., Egedal, J., Fox, W., Le, A., Vrublevskis, A., and Bonde, J.: Experimental investigation of the trigger problem in magnetic reconnection, Phys. Plasmas, 18, 055707, doi:10.1063/1.3562937, 2011.

Lapenta, G., Krauss-Varban, D., Karimabadi, H., Huba, J. D., Rudakov, L. I., , and Ricci, P.: Kinetic simulations of x-line expansion in 3-D reconnection, Geophys. Res. Lett., 33, L10102, doi:10.1029/2005GL025124, 2006.

Lau, Y.-T. and Finn, J. M.: 3-D kinematic reconnection in the presence of field nulls and closed field lines, Astrophys. J., 350, 672691, 1990.

Lawrence, E. E. and Gekelman, W.: Identification of a Quasiseparatrix Layer in a Reconnecting Laboratory Magnetoplasma, Phys. Rev. Lett., 103, 105002, doi:10.1103/PhysRevLett.103.105002, 2009.

Linton, M. G., Dahlburg, R. B., and Antiochos, S. K.: Reconnection of twisted flux tubes as a function of contact angle, Astrophys. J., 553, 905-921, 2001.

Loureiro, N. F., Cowley, S. C., Dorland, W. D., Haines, M. G., and Schekochihin, A. A.: X-point Collapse and Saturation in the Nonlinear Tearing Mode Reconnection, Phys. Rev. Lett., 95, 235003, doi:10.1103/PhysRevLett.95.235003, 2005.

Lowrie, W., Lukin, V. S., and Shumlak, U.: A priori mesh quality metric error analysis applied to a high-order finite element method, J. Comput. Phys., 230, 5564-5586, 2011.

Lukin, V. S.: Computational study of the internal kink mode evolution and associated magnetic reconnection phenomena, Ph.D. thesis, Princeton University, 2008.

Lukin, V. S., Glasser, A. H., Lowrie, W., and Meier, E. T.: Overview of $\mathrm{HiFi}$ - implicit spectral element code framework for general multi-fluid applications, in preparation, 2011.

Maclean, R. C., Parnell, C. E., and Galsgaard, K.: Is Null-Point Reconnection Important for Solar Flux Emergence?, Sol. Phys., 260, 299-320, 2009.

Masson, S., Pariat, E., Aulanier, G., and Schrijver, C. J.: The nature of flare ribbons in coronal null-point topology, Astrophys. 
J., 700, 559-578, 2009.

Meier, E. T., Lukin, V. S., and Shumlak, U.: Spectral element spatial discretization error in solving highly anisotropic heat conduction equation, Comp. Phys. Comm., 181, 837-841, 2010.

Munsat, T., Park, H. K., Classen, I. G. J., Domier, C. W., Donné, A. J. H., Luhmann Jr, N. C., Mazzucato, E., van de Pol, M. J., and the TEXTOR team: Localization of the magnetic reconnection zone during sawtooth crashes in tokamak plasmas, Nucl. Fusion, 47, L31-L35, 2007.

Parker, E. N.: Sweet's mechanism for merging magnetic fields in conducting fluids, J. Geophys. Res., 62, 509-520, 1957.

Pontin, D. I.: Three-dimensional magnetic reconnection regimes: A review, Adv. Space Res., 47, 1508-1522, 2011.

Pontin, D. I. and Galsgaard, K.: Current amplification and magnetic reconnection at a three-dimensional null point: Physical characteristics, J. Geophys. Res., 112, A03103, doi:10.1029/2006JA011848, 2007.

Pontin, D. I., Hornig, G., and Priest, E. R.: Kinematic reconnection at a magnetic null point: fan-aligned current, Geophys. Astro. Fluid, 99, 77-93, 2005.

Pontin, D. I., Bhattacharjee, A., and Galsgaard, K.: Current sheet formation and nonideal behavior at three-dimensional magnetic null points, Phys. Plasmas, 14, 052106, doi:10.1063/1.2722300, 2007.

Porcelli, F.: Collisionless m=1 Tearing Mode, Phys. Rev. Lett., 66, 425-428, 1991.

Priest, E. R. and Pontin, D. I.: Three-dimensional null point reconnection regimes, Phys. Plasmas, 16, 122101, doi:10.1063/1.3257901, 2009.

Priest, E. R. and Titov, V. S.: Magnetic reconnection at threedimensional null points, Philos. Trans. R. Soc. London, Ser. A, 354, 2951-2992, 1996.
Qiu, J.: Observational analysis of magnetic reconnection sequence, Astrophys. J., 692, 1110-1124, 2009.

Rosenbluth, M. N., Dagazian, R. Y., and Rutherford, P. H.: Nonlinear properties of the internal $\mathrm{m}=1$ kink instability in the cylindrical tokamak, Phys. Fluids, 16, 1894-1902, 1973.

Schindler, K., Hesse, M., and Birn, J.: General Magnetic Reconnection, Parallel Electric Fields, and Helicity, J. Geophys. Res., 93, 5547-5557, 1988.

Simakov, A. N. and Chacón, L.: Quantitative, comprehensive, analytical model for magnetic reconnection in Hall magnetohydrodynamics, Phys. Rev. Lett., 101, 105003, doi:10.1103/PhysRevLett.101.105003, 2008.

Sweet, P. A.: Electromagnetic Phenomena in Cosmical Physics, chap. The Neutral Point Theory of Solar Flares, 123-134, Cambridge U.P., New York, 1958.

Taylor, J. B.: Relaxation of toroidal plasma and generation of reverse magnetic fields, Phys. Rev. Lett., 33, 1139-1141, 1974.

Terasawa, T.: Hall current effect on tearing mode instability, Geophys. Res. Lett., 10, 475-478, 1983.

Woltjer, L.: A theorem on force-free magnetic fields, Proc. Natl. Acad. Sci. USA, 44, 489-491, 1958.

Xiao, C. J., Wang, X. G., Pu, Z. Y., Zhao, H., Wang, J. X., Ma, Z. W., Fu, S. Y., Kivelson, M. G., Liu, Z. X., Zong, Q. G., Glassmeier, K. H., Balogh, A., Korth, A., Reme, H., and Escoubet, C. P.: In situ evidence for the structure of the magnetic null in a 3-D reconnection event in the Earth's magnetotail., Nat. Phys., 2, 478-483, 2006.

Zweibel, E. G. and Yamada, M.: Magnetic Reconnection in Astrophysical and Laboratory Plasmas, Annu. Rev. Astron. Astr., 47, 291-332, 2009. 